\title{
Preface to the special issue: Macrocyclic and supramolecular chemistry
}

The 14th Conference of Macrocyclic Chemistry and the 6th Conference of Supramolecular Chemistry was held in Northwest Normal University in Lanzhou, Gansu Province, China, from 11 to 13 August, 2008. More than 200 participants from 40 universities and institutes discussed the development of macrocyclic and supramolecular chemistry in China. More than 50 plenary and oral presentations concerning the synthesis, recognition, assembly and functions of macrocyclic and supramolecular systems were held, presenting significant achievements in these areas. The participants enjoyed not only the lectures about the latest advances in macrocyclic and supramolecular chemistry but also stimulating talks and meaningful discussions.

Twenty-six years after the First Conference of Macrocyclic Chemistry was held in Lanzhou in 1982, it was held again in Lanzhou, emphasizing progress in China's macrocyclic and supramolecular chemistry. The First Conference of Supramolecular Chemistry was held in Tianjin in 1998, which initiated a time of increasing research by distinguished scientists in various facets of these two important sub-fields of chemistry. Younger researchers have built on the foundations of their predecessors, making steady research progress in both macrocyclic and supramolecular chemistry. The combined efforts of experienced and recently trained professional chemistry researchers hold out substantial promise for future progress in understanding macrocyclic and su- pramolecular chemistry.

More than 300 papers were submitted to this conference. In order to fully present these research achievements, three special issues (two in Chinese and one in English) of Macrocyclic and Supramolecular Chemistry will be published in Science in China Series BChemistry. As the chairman of the conference, I was invited to be the guest editor of these special issues. I hope that the publishing of these special issues will be helpful to chemists who are interested in macrocyclic and supramolecular chemistry. I would like to express my sincere appreciation to all of the contributors and to the reviewers of the special issues as well as to the editorial staff of Science in China Series B-Chemistry, especially ZHU XiaoWen, LI ZhanYing, and FANG Chen. Finally, I am very appreciated to all the chemistry researchers who delivered lectures, and to all of the participants who made the 14th Conference of Macrocyclic Chemistry and the 6th Conference of Supramolecular Chemistry a highly successful event.

Professor LIU Yu

Department of Chemistry

Nankai University, Tianjin

March, 2009

doi: 10.1007/s11426-009-0120-1 